\title{
Network analysis of anxiety and depressive symptoms among quarantined individuals: cross-sectional study
}

\author{
Mustafa Abdul Karim, Sami Ouanes, Shuja M. Reagu and Majid Alabdulla
}

\section{Background}

The mental health burden of COVID-19 has been examined in different settings. Existing research has relied on the latent variable model in assessing COVID-19-related distress. Network theory provides an alternative framework wherein symptoms are conceptualised as causal, interconnected constituents rather than outcomes of mental disorders.

\section{Aims \\ To assess networks of self-reported anxiety and depressive symptoms among quarantined individuals.}

\section{Method}

Consenting individuals in different quarantine centres in Qatar completed the Patient Health Questionnaire Anxiety and Depression Scale. We used partial correlation network methods to illustrate interactions of self-reported psychopathology.

\section{Results}

Participants with COVID-19 were significantly older and had a significantly higher proportion of males. The most central node was COVID-19, followed by thoughts of self-harm. COVID-19 status was strongly positively connected to thoughts of self-harm, which was positively connected to psychomotor changes, which were connected to decreased concentration. COVID-19 status was also positively connected to feeling anxious, which was strongly connected to inability to concentrate, which was connected to feeling afraid.

\section{Conclusions}

COVID-19 was the most influential factor, with the highest number and strength of connections to psychopathology in a network of anxiety and depressive symptoms in a quarantine setting. Beyond the resolution of the infection, therapeutic interventions targeting psychomotor changes might prove beneficial in reducing suicidality among quarantined individuals with COVID-19. Follow-up with mental health services after COVID-19 infection is needed to restore psychological well-being. Further research is needed to understand the short- and long-term psychological effects of COVID-19, and the outcomes of different therapeutic interventions.

\section{Keywords}

Depressive disorders; anxiety disorders; network analysis; pandemic; COVID-19.

\section{Copyright and usage}

(C) The Author(s), 2021. Published by Cambridge University Press on behalf of the Royal College of Psychiatrists. This is an Open Access article, distributed under the terms of the Creative commons Attribution licence (https://creativecommons.org/ licenses/by/4.0/), which permits unrestricted re-use, distribution, and reproduction in any medium, provided the original work is properly cited.
Following its outbreak in the Wuhan province of China in December 2019, the emergence and rapid spread of COVID-19, caused by severe acute respiratory syndrome coronavirus 2 (SARS-CoV-2), was declared a pandemic by the World Health Organization on 11 March 2020. ${ }^{1}$ Nations worldwide have been affected, with devastating socioeconomic and mental health outcomes. $^{2}$ Neuropsychiatric sequelae of COVID-19 have been reported, ${ }^{3}$ and the neurotropic effects of the virus and host immunologic response are yet to be fully understood. ${ }^{4}$

Most studies focused on the indirect psychological impact of the pandemic on non-infected individuals, with a few addressing psychiatric manifestations in patients testing positive for SARSCoV-2. ${ }^{5}$ Bo et al's cross-sectional study of 714 clinically stable patients with COVID-19 found a $96.2 \%$ prevalence of significant post-traumatic stress symptoms associated with the disease $(95 \%$ CI 94.8-97.6\%). ${ }^{6}$ Zhang et al screened for psychological distress among patients newly recovered from COVID-19 compared with individuals under quarantine and the general population. Using the Patient Health Questionnaire (PHQ-9) and seven-item Generalized Anxiety Scale (GAD-7), with a cut-off score of 10 or above for both scales, the prevalence of depression among individuals with COVID-19 was found to be $29.2 \%$, significantly higher than other groups $(P=0.016)$. Rates of anxiety or depression comorbid with anxiety did not reach statistical significance. ${ }^{7}$ However, analysing survey outcomes by using similar diagnostic thresholds and summary scores might hinder scientific progress in understanding and treating psychopathology. ${ }^{8}$

\section{Network analysis and psychopathology}

Statistical manuals and classification systems interpret psychopathology by using the disease model, whereby clinical symptoms represent manifestations of a latent variable (i.e mental disorders) and share a causal background. Psychometrics aggregate outcomes in a total score that presumably reflects the severity of the latent variable. The DSM-5 has been criticised for its selective reliance on expert opinion in defining mental illness notwithstanding existing clinical research, ${ }^{9}$ heralding the National Institute of Mental Health (NIMH)'s decision to halt funding for research predicated on DSM diagnosis in favour of the more scientifically based Research Domain Criteria. ${ }^{10}$ Furthermore, the constellation of symptoms defining a mental disorder is amassed irrespective of potential stressors or comorbid conditions, with no reference to differential weight or significance of set components. For instance, excessive anxiety and worry that is difficult to control, and the presence of low mood, loss of interest or pleasure, are contextually blind prerequisites for the diagnoses of generalised anxiety disorder and major depressive disorder, respectively. ${ }^{11}$ Said core symptoms might not consistently represent the most central or relevant sources of distress for the population of interest. Cramer et al intriguingly tackled this limitation by assessing patterns of depressive symptoms following four stressful life events: stress (owing to work, finances, etc), romantic loss, health problems and interpersonal conflict. Decreased appetite was found to be highly central in the conflict group compared with loss of interest in those who experienced romantic loss. Additionally, correlation patterns 
between depressive symptoms differed among groups, as the association between depressed mood and thoughts of death was stronger in cohorts experiencing health problems and interpersonal conflicts. In contrast, strong connections between worthlessness and thoughts of death was more pronounced in groups experiencing stress or romantic loss. ${ }^{12}$ More recently, McWilliams et al analysed selfreported depressive symptoms in patients seeking treatment for chronic pain, and found difficulty concentrating, loss of interest or pleasure, depressed mood and fatigue to be the most central symptoms. ${ }^{13}$

Such conceptualisation of psychopathology is possible through the application of network theory, wherein mental disorders are conceived as systems of interconnected variables. This statistical framework allows for in-depth analysis of centrality and connectivity among different components of mental disorders, without assuming a common underlying biological aetiology or equivalent diagnostic weight for clinical symptoms. Psychopathology is therefore construed as a constituent of mental disorders rather than its outcome or reflection. ${ }^{14}$ Further exploration is needed to comprehend the psychological ramifications of the current pandemic, and applying network theory offers a more extensive appreciation of presenting symptoms. We have previously assessed the psychological outcome of COVID-19 in quarantine settings, and found that quarantined participants who were positive for COVID-19 reported significantly higher anxiety and depressive symptoms compared with controls (quarantined participants without COVID-19). ${ }^{15}$ In this study, we aim to perform an exploratory network analysis of data from our previous study, with the goal of providing a deeper understanding of psychopathology and generating foci for therapeutic intervention and future research.

\section{Method}

\section{Participants and procedures}

We carried out a network analysis using data from our nationwide study examining the prevalence of depressive and anxiety symptoms in individuals quarantined for COVID-19 in Qatar. ${ }^{15}$ Inclusion criteria comprised all adults aged 18 or above who were located in quarantine cites. Those unable to consent to participation because of illiteracy, health conditions or other factors were deemed ineligible to take part in the study. Participant selection was randomised based on room number allocation: alternate room numbers in smaller facilities and every fifth room in larger facilities. We performed a network analysis on depressive and anxiety symptoms in participants with COVID-19 (positive COVID-19 polymerase chain reaction (PCR) test). Quarantined participants with a negative COVID-19 PCR test served as controls. All individuals with pending or inconclusive COVID-19 PCR test results were excluded. This study is not a trial and has not been registered under any registry. It only received approval from IRB at Hamad Medical Corporation (MRC-05-086) and is in accordance with the World Medical Association's Declaration of Helsinki. All participants provided written informed consent to enrol in the study and approved the use of collected data for research purposes.

\section{Measures}

Demographic information and COVID-19 status were collected in the first part of the study questionnaire. The second part comprised the Patient Health Questionnaire Anxiety and Depression Scale, a reliable tool for the composite measure of depressive and anxiety symptoms in different settings, including both the PHQ-9 and GAD-7. ${ }^{16}$ It was chosen as our screening tool given the availability of validated Arabic versions of both the PHQ-9 and GAD-7. ${ }^{17}$

\section{Network analysis}

We performed a partial correlation network study by using the EstimateNetwork feature of the R package 'bootnet' (R 4.0.5 for Windows, "The Foundation for Statistical Computing", Vienna, Austria; see https://www.r-project.org/). JASP software (JASP 0.14 for Windows, JASP Team, Amsterdam, the Netherlands; see https://jasp-stats.org/) was used to produce plots. The nodes of each network corresponded to the list of depressive and anxiety symptoms as per the PHQ-9 and GAD-7, respectively. To examine the links between COVID-19 and depressive and anxiety symptoms, we also chose to add COVID-19 status (as per the PCR result) as a node in the network. We chose this approach previously followed by Fried et a ${ }^{18}$ instead of establishing two different networks for COVID-19-positive and COVID-19-negative groups, to avoid Berkson's bias. We used the Extended Bayesian Information Criterion Graphical Least Absolute Shrinkage and Selection Operator (EBICglasso) regularised partial correlation estimator. We calculated the matrix of partial correlations between symptoms in each study group and proceeded with the graphical representation of the symptom network in each of the groups. To plot the network, we chose the following options: tuning parameter $(\gamma)=0.5$, cut-off of 0.1 , minimum value of 0 and maximum value of 1 .

We also calculated the following centrality indices for the two networks estimated jointly: strength of the node (i.e. the sum of all edges of a given node to all other nodes) ${ }^{19}$ and Eigenvector centrality (i.e. the degree to which a node is connected to other central nodes). Other centrality indices such as betweenness and closeness were not used because of their poor stability. ${ }^{20}$

We examined the stability of the network by assessing the stability of edge weights and the stability of centrality indices. ${ }^{21}$ First, we determined the $95 \%$ confidence intervals for each edge, using 1000 bootstraps. We also estimated the edge stability coefficient, which represents the maximum proportion of the sample that can be dropped to retain a correlation of at least 0.7 between the original edge weights and the edge weights in the bootstrapped data-sets, with $95 \%$ probability. Second, we calculated the centrality stability coefficient, which corresponds to the maximum proportion of the sample that can be dropped to retain a correlation of at least 0.7 between the original edge weights and the edge weights in the bootstrapped data-sets, with $95 \%$ probability. ${ }^{22}$

\section{Results}

\section{General characteristics}

A total of 748 individuals participated in the study. Individuals with an inconclusive COVID-19 PCR test or those for whom the test result was not available at the time of survey distribution were excluded. Our sample comprised 394 participants with a positive COVID-19 PCR and 269 controls (with a negative COVID-19 PCR) (Fig. 1). COVID-19 participants were significantly older $(41.4 \pm 12.6 v .35 .2 \pm 10.5$ years; $P<0.001)$ and had a significantly higher percentage of males (66.8\% v. 34.2\%; $P<0.001)$.

\section{Network analysis}

The network analysis is illustrated in Figure 2. The centrality plots for each node are shown in Figure 3. Of all possible 136 edges in the COVID-19 network, 107 (78.7\%) were estimated to be above zero.

Figure 4 shows the bootstrapped 95\% confidence intervals for each edge. There are no clear cut-offs yet to interpret these confidence intervals, and so their interpretation can be difficult. In our model, the $95 \%$ confidence intervals seem to be rather large and 


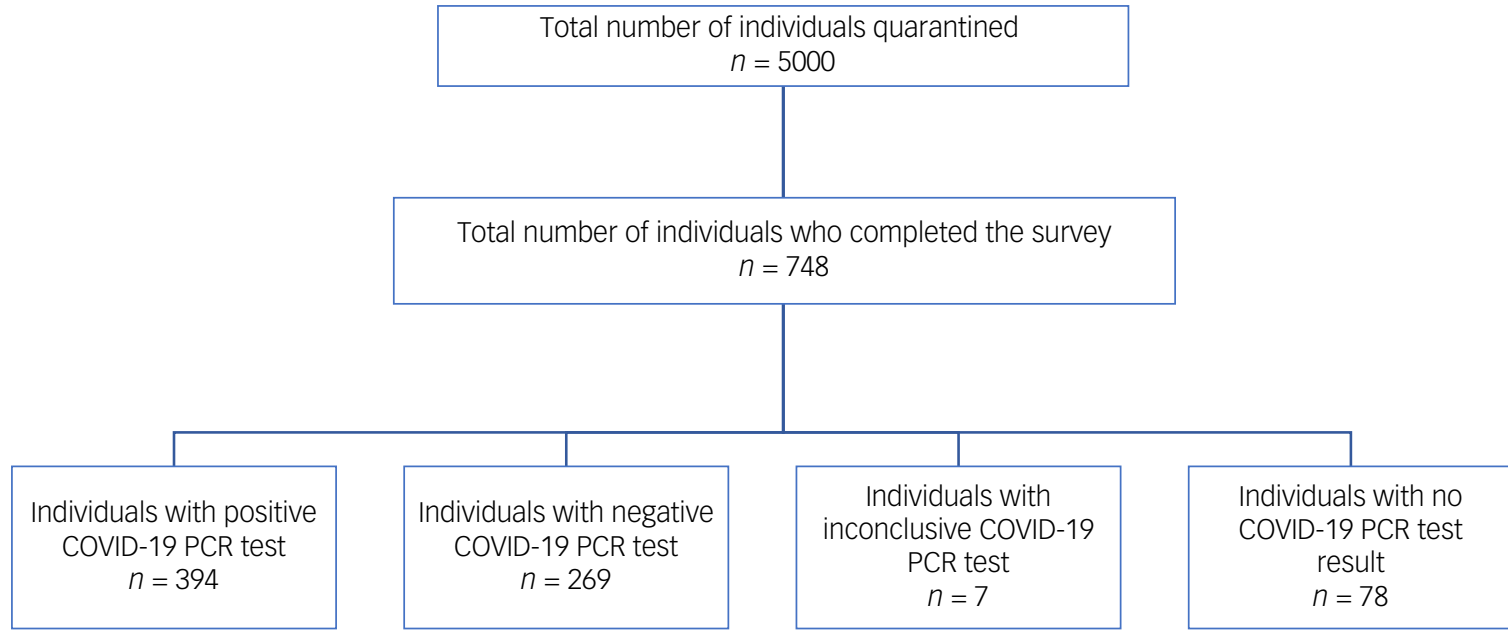

Total number of individuals included $n=663$

Total number of individuals excluded $n=85$

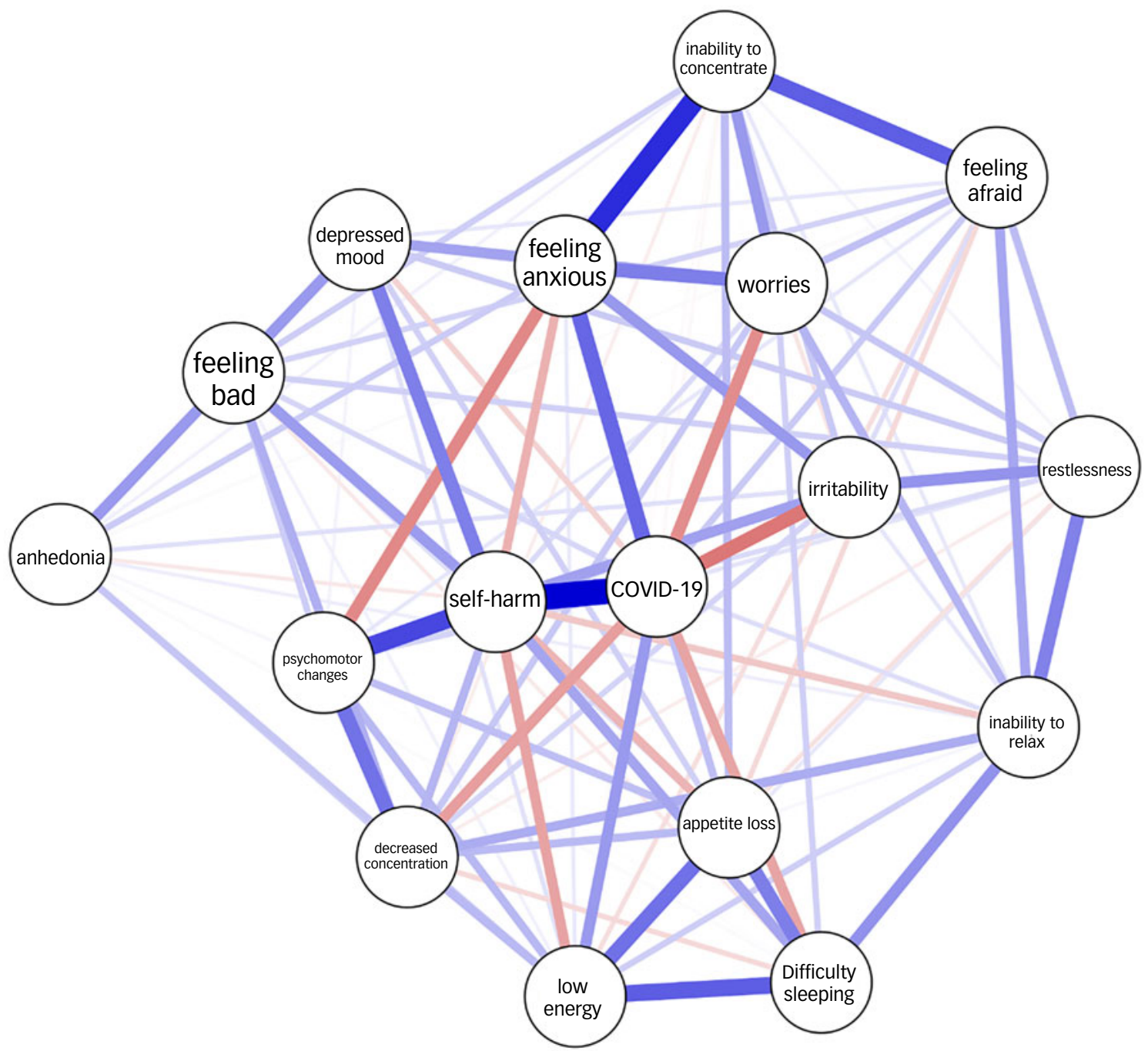



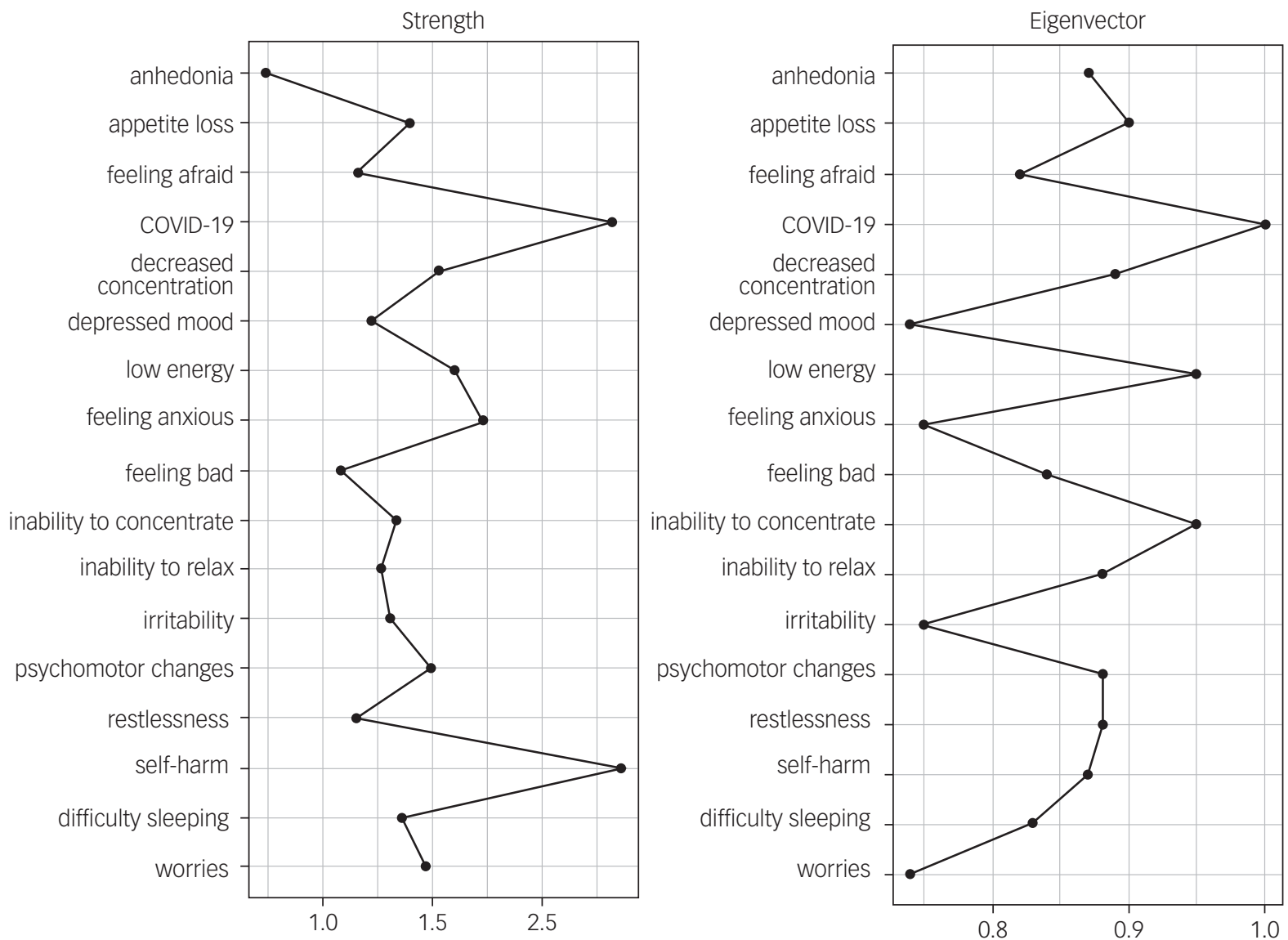

Fig. 3 Centrality plots for the network of depressive and anxiety symptoms in relation to COVID-19 status. COVID-19 refers to CoVID-19 status (positive polymerase chain reaction test result).

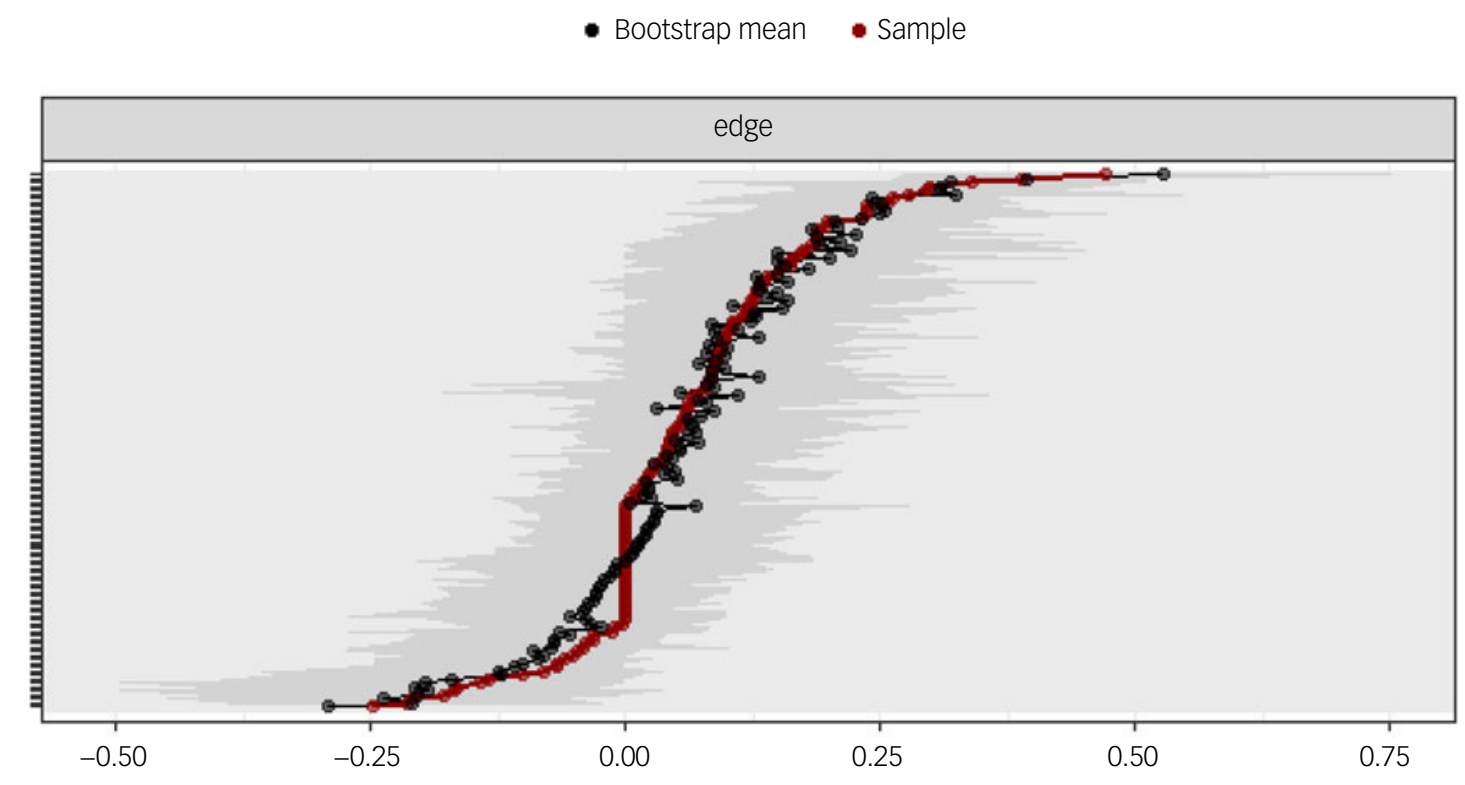

Fig. 4 Network edge weight stability of depressive and anxiety symptoms in relation to COVID-19 status. Edge weights are indicated by a solid red line. The $95 \%$ confidence intervals around these edge weights are represented in grey. The bootstrapped mean $95 \%$ confidence intervals are indicated by a black line. 


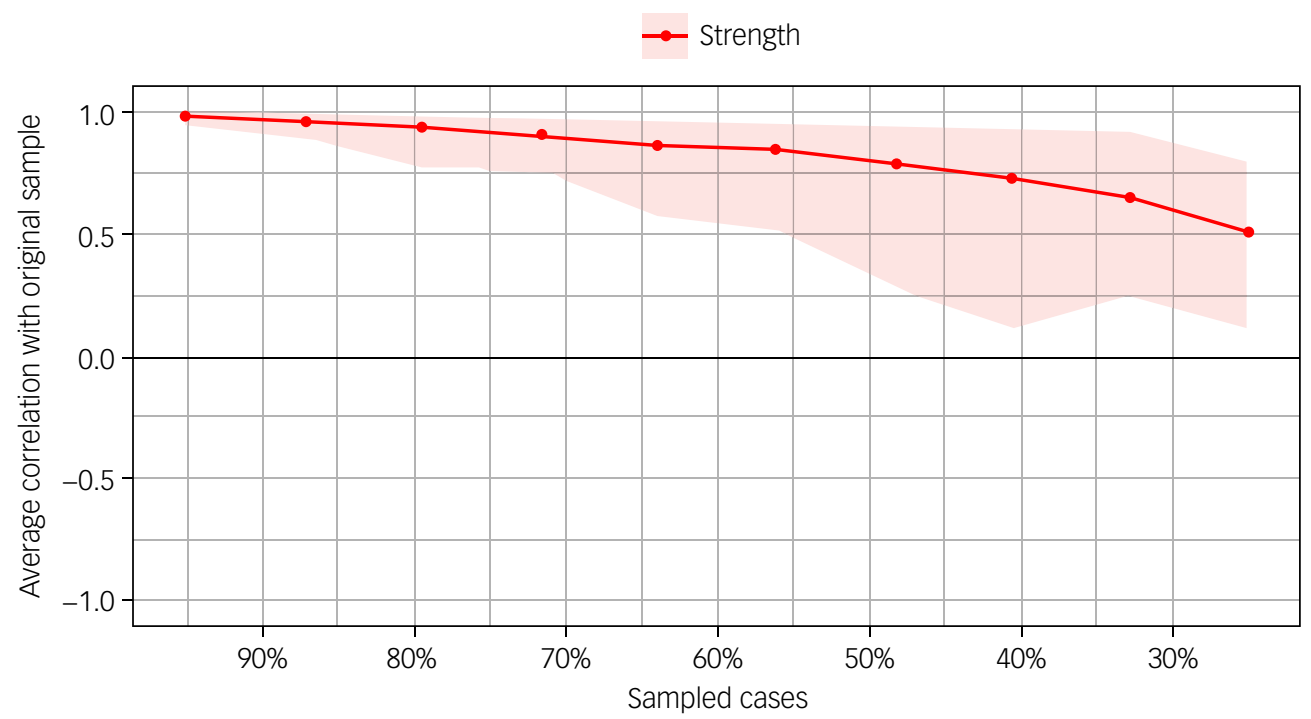

Fig. 5 Correlations of the centrality of nodes in the original network with the centrality of bootstrapped networks sampled while dropping participants.

overlapping, which means that the order of the edge weights can only be interpreted with caution.

The edge stability coefficient was 0.594 , which can be interpreted as very good ${ }^{22}$. In addition, Figure 5 shows correlations of the centrality of nodes in the original network with the centrality of bootstrapped networks sampled while dropping participants. The centrality stability coefficient was 0.36 , which can be interpreted as acceptable. $^{22}$

The most central node was COVID-19 status, followed by thoughts of self-harm (TSH). COVID-19 status was strongly positively connected to TSH, which in turn was positively connected to psychomotor changes (PMCs). PMCs were connected to decreased concentration. COVID-19 status was also positively connected to feeling anxious, which was strongly connected to inability to concentrate, which in turn was connected to feeling afraid. In addition, COVID-19 status was negatively associated with irritability, worries and decreased concentration (Table 1).

\section{Discussion}

We analysed our data with a $\gamma$ of 0.5 to balance revealing a true network structure and excluding spurious relationships. The choice of tuning parameter setting was based on the work by Epskamp, who found the adoption of a $\gamma$ of 0.75 or 1.00 to be non-superior to $\gamma=0.5$ in an established data-set. ${ }^{23}$ The accuracy of centrality indices in our network is acceptable (centrality stability coefficient of 0.36 ), as values above 0.25 are suggested to reflect accuracy, with a preference for values above $0.5{ }^{24}$ The edge stability coefficient indicates a very good accuracy level of depicted connections. However, because of large, overlapping bootstrapped 95\% confidence intervals, the order of the edge weights needs to be interpreted with caution. Based on these findings, the elucidated network represents a relatively valid tool in interpreting the covariance and connections of psychopathology in quarantine settings.

In accordance with centrality indices, COVID-19 status had the highest degree and strength of connectivity to anxiety and depressive symptoms. Although our network does not reveal the direction of connections, it still emphasises the central role of COVID-19 in propagating psychopathology. The strongest connection to COVID-19 status was TSH (weighted matrix of 0.471). In
2020, several reports shed light on suicide cases during the pandemic. ${ }^{25-27}$ Despite warnings of a concurrent suicide pandemic, subsequent analyses of national and regional statistics revealed either no significant change or a reduction in suicide rates during the COVID-19 period. No evidence of increase in suicide rates was found in a time-series analysis of data from 21 countries. $^{28}$ Compared with the same period in the preceding 14 years, Deisenhammer and Kemmler found a significant decrease in suicide numbers between April and September of 2020 in the state of Tyrol, Austria. ${ }^{29}$ In New York, no significant change was observed in suicidal ideation, self-injurious behaviour and suicide attempts among patients requiring emergent psychiatric evaluation. A significant decline was also noted in suicide attempts among children and adolescents during the pandemic compared with the preCOVID period $(1.5$ v. $5 \% ; P<0.001)$. Furthermore, patients with COVID-19 were less likely to be depressed or report suicidal ideation compared with those without COVID-19. ${ }^{30}$

Based on our previous study, the majority of our participants were migrant workers, and participants with COVID-19 had higher depressive (PHQ-9 score 6.1 v. $2.8 ; P<0.001$ ) and anxiety scores (GAD-7 score 4.8 v. $2.3 ; P<0.001$ ) compared with controls. ${ }^{15}$ In addition, our participants comprised quarantined individuals. Social isolation and loneliness in the context of quarantine can lead to symptoms of depression, anxiety and post-traumatic stress disorders. ${ }^{31}$

Hou et al showed that COVID-19 infection risk was positively associated with depressive symptoms among home quarantined young adults, and that this relationship was moderated by social support. $^{32}$ In Germany, the subjectively assumed stay-at-home order and higher levels of restrictions during lockdown were associated with poorer mental health. ${ }^{33}$ Furthermore, Jassim et al's cross-sectional study revealed that quarantine and isolation as a result of COVID-19 were associated with clinically significant depression, post-traumatic stress and perceived stigma. $^{34}$ Quarantine and social isolation might have contributed to the strong positive connection between COVID-19 status and TSH, in contrast to other studies where participants mainly included unquarantined native populations.

Our network revealed a strong connection between TSH and PMCs, and therapeutic interventions targeting PMCs might be beneficial in reducing suicidality among quarantined individuals 


\begin{tabular}{|c|c|c|c|c|c|c|c|c|c|c|c|c|c|c|c|c|c|}
\hline \multirow[b]{2}{*}{ Variable } & \multicolumn{17}{|c|}{ Network } \\
\hline & $\begin{array}{l}\text { COVID- } \\
19\end{array}$ & $\begin{array}{l}\text { Feeling } \\
\text { anxious }\end{array}$ & $\begin{array}{l}\text { Inability to } \\
\text { concentrate }\end{array}$ & Worries & $\begin{array}{l}\text { Inability } \\
\text { to relax }\end{array}$ & Restlessness & Irritability & $\begin{array}{l}\text { Feeling } \\
\text { afraid }\end{array}$ & Anhedonia & $\begin{array}{l}\text { Depressed } \\
\text { mood }\end{array}$ & $\begin{array}{l}\text { Difficulty } \\
\text { sleeping }\end{array}$ & $\begin{array}{l}\text { Low } \\
\text { energy }\end{array}$ & $\begin{array}{l}\text { Appetite } \\
\text { loss }\end{array}$ & $\begin{array}{c}\text { Feeling } \\
\text { bad }\end{array}$ & $\begin{array}{l}\text { Decreased } \\
\text { concentration }\end{array}$ & $\begin{array}{l}\text { Psychomotor } \\
\text { changes }\end{array}$ & $\begin{array}{l}\text { Self- } \\
\text { harm }\end{array}$ \\
\hline COVID-19 & 0.000 & 0.278 & -0.015 & -0.208 & 0.058 & 0.051 & -0.249 & 0.098 & 0.000 & -0.081 & -0.167 & 0.187 & 0.118 & 0.074 & -0.178 & 0.078 & 0.471 \\
\hline Feeling anxious & 0.278 & 0.000 & 0.389 & 0.236 & 0.002 & 0.000 & 0.188 & 0.000 & 0.094 & 0.084 & 0.000 & 0.038 & 0.061 & 0.000 & 0.000 & -0.216 & -0.142 \\
\hline $\begin{array}{l}\text { Inability to } \\
\text { concentrate }\end{array}$ & -0.015 & 0.389 & 0.000 & 0.188 & 0.040 & 0.019 & 0.100 & 0.296 & 0.026 & 0.000 & -0.003 & -0.010 & 0.122 & 0.090 & -0.032 & -0.002 & 0.000 \\
\hline Worries & -0.208 & 0.236 & 0.188 & 0.000 & 0.137 & 0.104 & -0.048 & 0.114 & 0.000 & 0.171 & 0.087 & 0.000 & 0.000 & 0.000 & 0.101 & 0.000 & 0.067 \\
\hline Inability to relax & 0.058 & 0.002 & 0.040 & 0.137 & 0.000 & 0.232 & 0.044 & 0.162 & 0.031 & 0.000 & 0.199 & 0.090 & 0.016 & 0.000 & 0.152 & 0.000 & -0.101 \\
\hline Restlessness & 0.051 & 0.000 & 0.019 & 0.104 & 0.232 & 0.000 & 0.197 & 0.122 & 0.056 & 0.096 & 0.000 & 0.040 & -0.054 & 0.086 & -0.033 & 0.066 & 0.000 \\
\hline Irritability & -0.249 & 0.188 & 0.100 & -0.048 & 0.044 & 0.197 & 0.000 & 0.061 & 0.038 & 0.000 & 0.000 & 0.000 & 0.000 & 0.000 & 0.078 & 0.127 & 0.174 \\
\hline Feeling afraid & 0.098 & 0.000 & 0.296 & 0.114 & 0.162 & 0.122 & 0.061 & 0.000 & 0.009 & 0.045 & 0.000 & -0.059 & -0.069 & 0.081 & 0.000 & 0.042 & 0.000 \\
\hline Anhedonia & 0.000 & 0.094 & 0.026 & 0.000 & 0.031 & 0.056 & 0.038 & 0.009 & 0.000 & 0.187 & 0.010 & 0.104 & 0.016 & 0.042 & 0.088 & 0.000 & -0.041 \\
\hline Depressed mood & -0.081 & 0.084 & 0.000 & 0.171 & 0.000 & 0.096 & 0.000 & 0.045 & 0.187 & 0.000 & 0.085 & 0.053 & 0.000 & 0.162 & 0.000 & 0.022 & 0.235 \\
\hline Difficulty sleeping & -0.167 & 0.000 & -0.003 & 0.087 & 0.199 & 0.000 & 0.000 & 0.000 & 0.010 & 0.085 & 0.000 & 0.298 & 0.237 & -0.039 & -0.069 & 0.004 & 0.165 \\
\hline Low energy & 0.187 & 0.038 & -0.010 & 0.000 & 0.090 & 0.040 & 0.000 & -0.059 & 0.104 & 0.053 & 0.298 & 0.000 & 0.263 & 0.025 & 0.127 & 0.130 & -0.168 \\
\hline Appetite loss & 0.118 & 0.061 & 0.122 & 0.000 & 0.016 & -0.054 & 0.000 & -0.069 & 0.016 & 0.000 & 0.237 & 0.263 & 0.000 & 0.058 & 0.126 & 0.117 & -0.136 \\
\hline Feeling bad & 0.074 & 0.000 & 0.090 & 0.000 & 0.000 & 0.086 & 0.000 & 0.081 & 0.042 & 0.162 & -0.039 & 0.025 & 0.058 & 0.000 & 0.148 & 0.092 & 0.182 \\
\hline $\begin{array}{l}\text { Decreased } \\
\text { concentration }\end{array}$ & -0.178 & 0.000 & -0.032 & 0.101 & 0.152 & -0.033 & 0.078 & 0.000 & 0.088 & 0.000 & -0.069 & 0.127 & 0.126 & 0.148 & 0.000 & 0.258 & 0.135 \\
\hline $\begin{array}{c}\text { Psychomotor } \\
\text { changes }\end{array}$ & 0.078 & -0.216 & -0.002 & 0.000 & 0.000 & 0.066 & 0.127 & 0.042 & 0.000 & 0.022 & 0.004 & 0.130 & 0.117 & 0.092 & 0.258 & 0.000 & 0.339 \\
\hline Self-harm & 0.471 & -0.142 & 0.000 & 0.067 & -0.101 & 0.000 & 0.174 & 0.000 & -0.041 & 0.235 & 0.165 & -0.168 & -0.136 & 0.182 & 0.135 & 0.339 & 0.000 \\
\hline
\end{tabular}


with COVID-19. Indic et al found an inverse relationship between participant motility and suicidal thinking in patients diagnosed with bipolar disorder and unipolar depression. ${ }^{35}$ A study of over 6000 Chinese women found that participants with major depression and a history of a suicide attempt reported more weight and appetite loss, insomnia and PMCs compared with women with depression and no prior suicide attempt. ${ }^{36}$ Rogers et al also found that agitation and nightmares significantly mediated the relationship between brooding and suicidal ideation. ${ }^{37}$ Furthermore, a retrospective analysis found higher levels of suicidal ideation among patients with agitated depression compared with controls (patients with non-agitated depression). However, this difference was non-significant after controlling for psychotic features. ${ }^{38}$ Conversely, Ballard et al showed that psychomotor agitation was not significantly elevated before suicidal behaviour. ${ }^{39}$ Among our participants, COVID-19 status and quarantine might have affected psychomotor activity and strengthened the relationship between TSH and PMCs.

PMCs was strongly connected to decreased concentration. COVID-19 status was also strongly connected with feeling anxious, which was in turn strongly connected to decreased concentration. On the periphery of the network, strong connections are additionally seen between difficulty sleeping, low energy and loss of appetite. The strong branching connections outside the direct impact of the most central node indicate the existence of a potentially reinforcing and self-sustaining relationship between anxiety and depressive symptoms beyond the effect of COVID-19. Hence, the resolution of infection might not be sufficient to ameliorate psychological distress. Li et al found that symptoms of insomnia, anxiety and depression significantly decreased after patients with COVID-19 were discharged to home isolation from quarantine facilities in Wuhan, China. However, out of 782 cases, 188 patients reported insomnia, 78 patients had anxiety symptoms and 84 patients reported depressive symptoms that persisted after discharge. ${ }^{40}$ A recent scoping review also found persistent physical and mental health problems and an overall lower quality of life up to 3 months after COVID-19 infection. ${ }^{41}$ Our findings, in accordance with existing literature, highlight the need for postinfection follow-up with mental health services in addressing psychological distress and restoring quality of life.

In quarantine settings, we found COVID-19 to be the most influential factor, with the highest number and strength of connections to psychopathology in a network of anxiety and depressive symptoms. Beyond the resolution of the infection, therapeutic interventions targeting PMCs might prove beneficial in reducing suicidality among quarantined individuals with COVID-19. The branching connections of psychopathology and relationships among anxiety and depressive symptoms beyond the direct effect of COVID-19 suggest the possibility of psychological distress propagation after COVID-19 infection, and emphasise the importance of follow-up with mental health services in restoring psychological well-being. Further research is needed to understand the short- and long-term psychological manifestations of COVID-19, and the outcomes of different management strategies in ameliorating psychopathology.

\section{Strengths and limitations}

We performed a network analysis of data obtained from a nationwide study involving randomly assigned participants in major quarantine cites in Qatar, stratified based on infection status. We were able to identify symptoms and factors of high centrality and explore the connections between anxiety and depressive symptoms. This approach is advantageous, offering a more detailed and comprehensive conceptualisation of mental distress compared with a proportional display of conjectured classification of psychopathology. However, our results could have been confounded by age and gender differences between cohorts. The lack of a third arm of controls, with and without COVID-19 outside quarantine facilities, obscured our ability to assess the impact of quarantine on mental health. In addition, the order of the edge weights in our model could only be interpreted with caution because of large, overlapping bootstrapped 95\% confidence intervals. Moreover, we did not include past psychiatric history in our analysis, which could have confounded our results. We were also unable to illustrate the directionality and possible causal relationship between symptoms, as our data was cross-sectional rather than longitudinal. Finally, it would have been ideal to evaluate anxiety and depressive symptoms by objective observation rather than self-reporting.

Mustafa Abdul Karim (D), Psychiatry Department, Hamad Medical Corporation, Qatar; and Weill Cornell Medicine, Qatar; Sami Ouanes, Psychiatry Department, Hamad Medical Corporation, Qatar; Shuja M. Reagu, Psychiatry Department, Hamad Medical Corporation, Qatar; Majid Alabdulla, Psychiatry Department, Hamad Medical Corporation, Qatar; and College of Medicine, Qatar University, Qatar

Correspondence: Mustafa Abdul Karim. Email: mkarim@hamad.qa

First received 23 Apr 2021, final revision 19 Oct 2021, accepted 27 Oct 2021

\section{Data availability}

The data that support the findings of this study are available from the corresponding author, M.A.K., upon reasonable request. The data are not publicly available due to restrictions from Hamad Medical Corporation.

\section{Author contributions}

All authors contributed to the conceptualisation, design and planning of the study. M.A.K. S.M.R. and M.A.A. wrote and revised the manuscript. S.O. conducted network analysis, and contributed to writing the results section and revising the manuscript.

\section{Funding}

This project did not receive funding from any governmental or private institution.

\section{Declaration of interest}

None.

\section{References}

1 World Health Organization (WHO). WHO Director-General's Opening Remarks at the Media Briefing on COVID-19. WHO, 2020 (https://reliefweb.int/report/ world/who-director-generals-opening-remarks-mission-briefing-covid-19-12march-2020?gclid=EAlaIQobChMImMLO_aSO9AIVnoxoCROSOWkUEAAYAYAA EgLnR_D_BWE).

2 Serafini G, Parmigiani B, Amerio A, Aguglia A, Sher L, Amore M. The psychological impact of COVID-19 on the mental health in the general population. QJM 2020; 113(8): 531-7.

3 Asadi-Pooya AA, Simani L. Central nervous system manifestations of COVID-19: a systematic review. J Neurol Sci 2020; 413: 116832.

4 Troyer EA, Kohn JN, Hong S. Are we facing a crashing wave of neuropsychiatric sequelae of COVID-19? Neuropsychiatric symptoms and potential immunologic mechanisms. Brain Behav Immun 2020; 87: 34-9.

5 Ren X, Huang W, Pan H, Huang T, Wang X, Ma Y. Mental health during the Covid19 outbreak in China: a meta-analysis. Psychiatr $Q$ 2020; 91: 1033-45.

6 Bo HX, Li W, Yang Y, Wang Y, Zhang Q, Cheung T, et al. Posttraumatic stress symptoms and attitude toward crisis mental health services among clinically stable patients with COVID-19 in China. Psychol Med 2021; 51(6): 1052-3.

7 Zhang J, Lu H, Zeng H, Zhang S, Du Q, Jiang T, et al. The differential psychological distress of populations affected by the COVID-19 pandemic. Brain Behav Immun 2020; 87: 49.

8 Borsboom D, Cramer AOJ. Network analysis: an integrative approach to the structure of psychopathology. Annu Rev Clin Psychol 2013; 9: 91-121.

9 Ghaemi SN. After the failure of DSM: clinical research on psychiatric diagnosis. World Psychiatry 2018; 17: 301-2. 
10 Winerman L. NIMH funding to shift away from DSM categories. Monit Psychol 2013; 44: 7.

11 American Psychiatric Association, DSM-5 Task Force. Diagnostic and Statistical Manual of Mental Disorders (5th edn). American Psychiatric Publishing, 2013.

12 Cramer AOJ, Borsboom D, Aggen SH, Kendler KS. The pathoplasticity of dysphoric episodes: differential impact of stressful life events on the pattern of depressive symptom inter-correlations. Psychol Med 2012; 42(5): 957-65.

13 McWilliams LA, Sarty G, Kowal J, Wilson KG. A network analysis of depressive symptoms in individuals seeking treatment for chronic pain. Clin J Pain 2017; 33(10): 899-904.

14 Contreras A, Nieto I, Valiente C, Espinosa R, Vazquez C. The study of psychopathology from the network analysis perspective: a systematic review. Psychother Psychosom 2019; 88: 71-83.

15 Reagu S, Wadoo O, Latoo J, Nelson D, Ouanes S, Masoodi N, et al. Psychological impact of the COVID-19 pandemic within institutional quarantine and isolation centres and its sociodemographic correlates in Qatar: a cross-sectional study. BMJ Open 2021; 11: e045794.

16 Kroenke K, Wu J, Yu Z, Bair MJ, Kean J, Stump T, et al. Patient Health Questionnaire anxiety and depression scale: initial validation in three clinical trials. Psychosom Med 2016; 78(6): 716-27.

17 AlHadi AN, AlAteeq DA, Al-Sharif E, Bawazeer HM, Alanazi H, AlShomrani AT, et al. An Arabic translation, reliability, and validation of Patient Health Questionnaire in a Saudi sample. Ann Gen Psychiatry 2017; 16(1): 32.

18 Fried El, Bockting C, Arjadi R, Borsboom D, Amshoff M, Cramer AOJ, et al. From Ioss to loneliness: the relationship between bereavement and depressive symptoms. J Abnorm Psychol 2015; 124(2): 256-65.

19 McNally RJ. Can network analysis transform psychopathology? Behav Res Ther 2016; 86: 95-104.

20 Bringmann LF, Elmer T, Epskamp S, Krause RW, Schoch D, Wichers M, et al. What do centrality measures measure in psychological networks? J Abnorm Psychol 2019; 128(8): 892-903.

21 Epskamp S, Borsboom D, Fried El. Estimating psychological networks and their accuracy: a tutorial paper. Behav Res Methods 2017; 50(1): 195-212.

22 Epskamp S, Fried El. Bootstrap Methods for Various Network Estimation Routines. Comprehensive R Archive Network, 2020 (https://cran.r-project. org/web/packages/bootnet/bootnet.pdf)

23 Epskamp S. Brief report on estimating regularized Gaussian networks from continuous and ordinal data. ArXiv [Preprint] 2016. Available from: https:// arxiv.org/abs/1606.05771v2.

24 Hevey D. Network analysis: a brief overview and tutorial. Heal Psychol Behav Med 2018; 6(1): 301

25 Syed NK, Griffiths MD. Nationwide suicides due to alcohol withdrawal symptoms during COVID-19 pandemic: a review of cases from media reports J Psychiatr Res 2020; 130: 289.

26 Rajkumar RP. Suicides related to the COVID-19 outbreak in India: a pilot study of media reports. Asian J Psychiatr 2020; 53: 102196.
27 Mamun MA, Griffiths MD. First COVID-19 suicide case in Bangladesh due to fear of COVID-19 and xenophobia: possible suicide prevention strategies. Asian J Psychiatr 2020; 51: 102073.

28 Pirkis J, John A, Shin S, DelPozo-Banos M, Arya V, Analuisa-Aguilar P, et al. Suicide trends in the early months of the COVID-19 pandemic: an interrupted time-series analysis of preliminary data from 21 countries. Lancet Psychiatry 2021; 8(7): 579-88.

29 Deisenhammer EA, Kemmler G. Decreased suicide numbers during the first 6 months of the COVID-19 pandemic. Psychiatry Res 2021; 295: 113623.

30 Ferrando SJ, Klepacz L, Lynch S, Shahar S, Dornbush R, Smiley A, et al. Psychiatric emergencies during the height of the COVID-19 pandemic in the suburban New York City area. J Psychiatr Res 2021; 136: 552.

31 Banerjee D, Rai M. Social isolation in Covid-19: the impact of loneliness. Int J SoC Psychiatry 2020; 66(6): 525.

32 Hou J, Yu Q, Lan X. COVID-19 infection risk and depressive symptoms among young adults during quarantine: the moderating role of grit and social support. Front Psychol 2021; 11: 577942.

33 Benke C, Autenrieth LK, Asselmann E, Pané-Farré CA. Lockdown, quarantine measures, and social distancing: associations with depression, anxiety and distress at the beginning of the COVID-19 pandemic among adults from Germany. Psychiatry Res 2020; 293: 113462.

34 Jassim G, Jameel M, Brennan E, Yusuf M, Hasan N, Alwatani Y. Psychological impact of COVID-19, isolation, and quarantine: a cross-sectional study. Neuropsychiatr Dis Treat 2021; 17: 1413-21.

35 Indic $P$, Murray G, Maggini C, Amore M, Meschi T, Borghi L, et al. Multi-scale motility amplitude associated with suicidal thoughts in major depression. PLOS One 2012; 7(6): e38761.

36 Zhu Y, Zhang H, Shi S, Gao J, Li Y, Tao M, et al. Suicidal risk factors of recurrent major depression in Han Chinese women. PLoS One 2013; 8(11): 80030.

37 Rogers ML, Schneider ME, Tucker RP, Law KC, Anestis MD, Joiner TE. Overarousal as a mechanism of the relation between rumination and suicidality. J Psychiatr Res 2017; 92: 31-7.

38 Olgiati $\mathrm{P}$, Serretti A, Colombo C. Retrospective analysis of psychomotor agitation, hypomanic symptoms, and suicidal ideation in unipolar depression. Depress Anxiety 2006; 23(7): 389-97.

39 Ballard ED, Vande Voort JL, Luckenbaugh DA, Machado-Vieira R, Tohen M, Zarate CA. Acute risk factors for suicide attempts and death: prospective findings from the STEP-BD study. Bipolar Disord 2016; 18(4): 363-72.

40 Li L, Wu MS, Tao J, Wang W, He J, Liu R, et al. A follow-up investigation of mental health among discharged COVID-19 patients in Wuhan, China. Front Public Health 2021; 9: 640352

41 Shanbehzadeh S, Tavahomi M, Zanjari N, Ebrahimi-Takamjani I, Amiri-Arimi S. Physical and mental health complications post-COVID-19: scoping review. J Psychosom Res 2021; 147: 110525. 